\title{
Highly Precise Modified Blue Whale Method Framed by Blending Bat and Local Search Algorithm for the Optimality of Image Fusion Algorithm
}

\author{
Sayantan Dutta \\ Department of Electronics and Telecommunication Engineering \\ Indian Institute of Engineering Science and Technology, Shibpur \\ Howrah-711103 \\ sayantan.dutta30@gmail.com
}

\author{
Ayan Banerjee \\ Department of Electronics and Telecommunication Engineering \\ Indian Institute of Engineering Science and Technology, Shibpur \\ Howrah-711103 \\ ayanb12@gmail.com
}

\begin{abstract}
Image fusion has gained huge popularity in the field of medical and satellite imaging for image analysis. The lack of usages of image fusion is due to a deficiency of suitable optimization techniques and dedicated hardware. In recent days WOA (whale optimization algorithm) is gaining popularity. Like another straightforward nature-inspired algorithm, WOA has some problems in its searching process. In this paper, we have tried to improve the WOA algorithm by modifying the WOA algorithm. This MWOA (modified whale optimization algorithm) algorithm is amalgamed with LSA (local search algorithm) and BA (bat algorithm). The LSA algorithm helps the system to be faster, and BA algorithm helps to increase the accuracy of the system. This optimization algorithm is checked using MATLAB R2018b. Simulated using ModelSim, and the synthesizing is done using Xilinx Vivado 18.2 synthesis tool. The outcome of the simulation result and the synthesis result outshine other metaheuristic optimization algorithms.
\end{abstract}

Keywords: WOA, modified WOA, prey, MWOA, BA, LSS, metaheuristic optimization, heuristic optimization.

\section{Introduction}

Image processing has vast applications in day to day life in the first world country, and its major field of application in medical imaging and satellite imaging. The precise, accurate, and reliable result is the key component of medical imaging. Generally, nature-inspired algorithms mimic the natural behavior of living creatures. Those natural techniques are used for clinical diagnosis in medical imaging. By using those natureinspired algorithms, we can find an accurate search result. One of such most popular metaheuristic algorithm is WOA, which is extensively used in image fusion. Like other metaheuristic algorithms, WOA has the same problem of premature convergence and trapping of local minima. To overcome such a problem, a modified WOA (MWOA) is proposed in this article. This MWOA is modified using LSA (local search algorithm) and BA (bat algorithm).

One of the newly developed metaheuristic algorithms is WOA. This algorithm is developed by Seyedali Mirjalili and his co-workers. The natural behavior of HW (humpback wheel) is mimicked in this WOA algorithm. The HW (humpback whale) hunts the prey is a group hunting process, but the problem is that it also traps in the same problem of local minima like other PSO (particle swarm optimization). The WOA algorithm can be divided into two parts, that is exploration and exploitation. The exploration part is controlled by controlling the distance, and the distance is controlled by the LSS (linear search strategy). In LSS, we use a parameter 'a' which use to control the distance, and it is the main cause that the WOA suffers in the trapping of local minima and premature convergence. This behavior of LSS influences convergence speed, accuracy, and reliability. To overcome those problems, we have modified the WOA algorithm. So, in this article, we have proposed MWOA (modified WOA) algorithm. In the WOA algorithm, the problem is in the searching process. To improve the searching process, we have introduced the LSA (local search algorithm), and this LSA algorithm is used when the unknown object is 
inside one unit radius or two-unit diameter. If the object is outside two-unit diameter, the BA (bat algorithm) is used for searching. In a dark environment, BA gives better efficiency to find the prey at the time of hunting. Actually, bats use the sound repulsion strategy in the searching process as well as to find the obstacle. When the object is very far from the searching probe, then the BA is used to control the velocity. On the other hand, if the object is closer, then it stops moving, and it gives an extra bit of protection to the unknown object. This type of essential feature is needed in medical imaging. At the end of the process, the result we have obtained will be accurate and precise.

Seyedali Mirjalili and his co-workers first proposed the WOA algorithm. After that, there are many nature-inspired algorithms that have been proposed, but the popularity of the WOA (whale optimization algorithm) remains the same. There are many modifications that happened upon whale optimization to improve performance, reliability, and computational speed. Some of the notable improvements are, 1) new operators are introduced in WOA to make the result more precise and reliable, 2) different parameters are added. So, that distance of exploration can be controlled more reliable way, 3) different searching techniques are added to extend the WOA. So, that a hybrid WOA can be made which can handle the more complex problem, 4) the Positional updations equation, which plays an important role in the convergence of WOA. This position update parameter is modified to achieve better convergence speed. Those different paths of modification of WOA are depicted below.

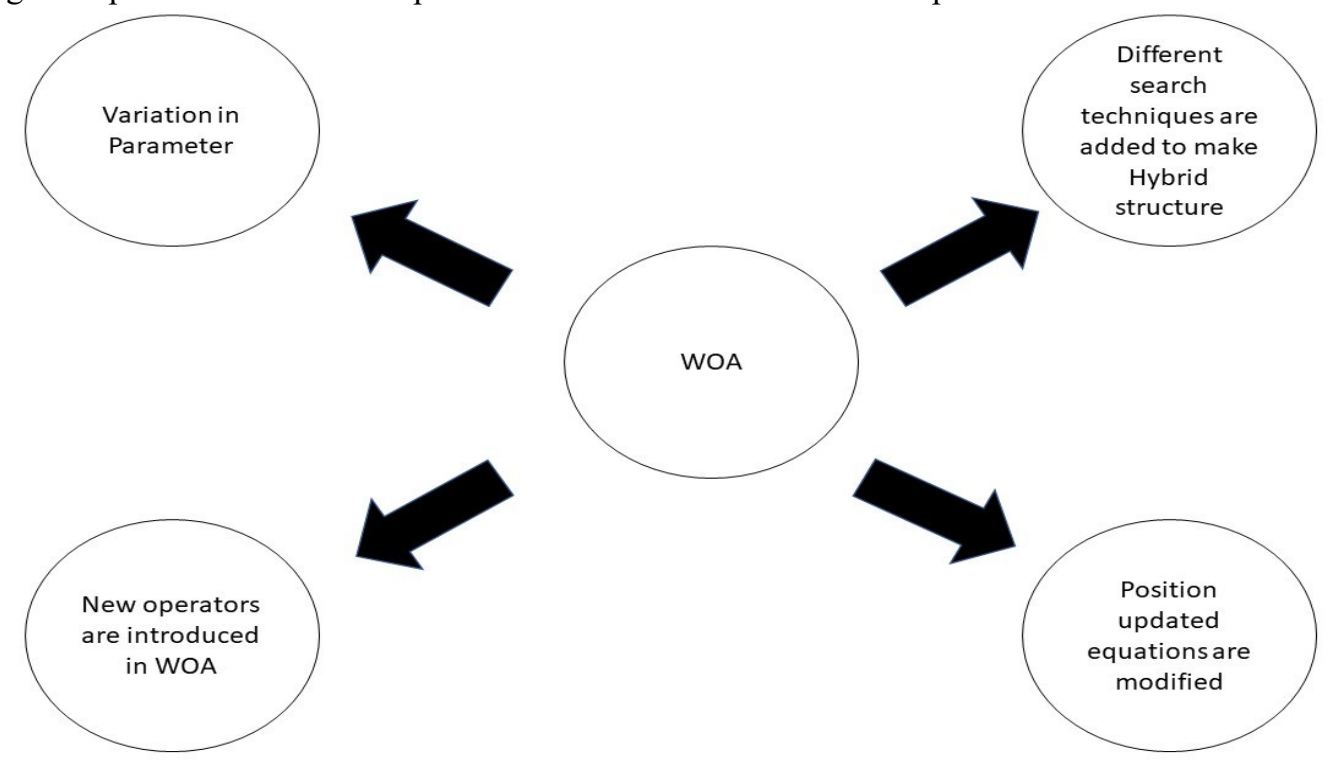

Figure 1. Different ways of modification of WOA in recent days

This article is organized in some sections and subsections. First, this paper is started with an introduction in section 1. In the second section, related work has been mentioned. In the next section (section 3), The theoretical background of our proposed work has been described. Whale optimization is described in subsection 3.1. The Bat algorithm is described in subsection 3.2. Followed by the Local Search algorithm is described in subsection 3.3. Then comes our proposed modified whale optimization algorithm, which has been discussed in detail in section 4. The experimental result is recorded in section 5. In the last section (section 6), a clear conclusion has been drawn on the supremacy of our MWOA.

\section{Related Works:}

In past years, many researchers have actively tried to refine the optimization process by using heuristic and metaheuristic algorithms. Whale optimization is one of the important and popular optimization techniques. The main problem of the WOA (whale optimization algorithm) algorithm is the searching mechanism. To overcome this problem, many researchers have tried to blend HM (heuristic method) with the MM (metaheuristic method) for finding the unknown object. This type of technique helps to improve the searching process. So, in this segment, recent application and trend of some metaheuristic optimization like BA (bat algorithm), WOA (whale optimization algorithm), and some blending of HOT (heuristic optimization technique) with WOA algorithm have been described.

Recent days COVID-19 virus spread worldwide and takes a lot of life. Many researchers have tried to find the COVID-19 positive patient by using X-ray image analysis. One of them is Mohamed Aqbdel-Basset and his co- 
Journal of Soft Computing Paradigm (JSCP) (2020)

Vol.02/ No.04

Pages: 195-208

http://irojournals.com/jscp/

DOI: https://doi.org/10.36548/jscp.2020.4.001

workers. It is very hard to find COVID-19 patients under the X-ray image. They also used the machine learning technique to find the condition of the patient. In this way, they have found in which stage the patient is. The patient may be noninfected, mild infected, or severely infected. They have taken the Xray image and specifies their region of interest in the image. This type of solution from the image is known as the ISP (image segmentation problem) problem. There are many techniques on ISP, but the most popular technique is thresholding. They have used a hybrid kind of thresholding by using WOA (whale optimization algorithm), which makes the process simple, accurate, and speedy [1].

The abnormally growing cells in the brain region are known as a brain tumor. It is very dangerous for patient life. If it can detect in an early stage, then it can be stopped from going to the next stage, and the dangerous effect can be reduced. The early stage of diagnosis can be done by a metaheuristic based optimization technique. Bo Yin and his co-workers have tried to detect brain tumors by using WOA (whale optimization algorithm). Their proposed work is divided into three phases that are background- removing, classification, and feature extraction. In their method, they modified the WOA by using LMT (logistic mapping technique) and CT (chaos theory). That helped them in classification stages and feature selection stages [2].

In the field of IDA (intelligent data analysis), optimization is an important technology. Before solving the analytical problem, the study of the optimization algorithm is needed. In recent years the development in the industry increases the complexity of the problem, and the traditional optimization method fails in this context. That makes new opportunities in the optimization field. The trend in recent days is to use MA (metaheuristic algorithm) algorithm. There are two reasons to use MA. One is easy implementation, and the second one is an outstanding searching capability. Naturally, MA becomes a good choice for solving OP (optimization problem). Jinkun Luo and his co-workers have proposed a hybrid optimization technique where they have used WOA (whale optimization algorithm), BA (bat algorithm), and OBL (opposition-based learning) [3].

In solving the problem of a diverse field in the real world, the PBMA (population-based metaheuristic algorithms) is a significant approach and dominates over the traditional approach. One of the recently popular SOBMA (swarm optimization based metaheuristic algorithms) is WOA (whale optimization algorithm). In WOA feeding strategy of HW (humpback wheel) is bubble net feeding. The WOA can solve a complex problem easily, but the problem is that it requires a huge amount of computation. Maryam AlJame and his co-workers proposed spark-WOA, which will reduce computational complexity and improve performance [4].

The WOA algorithm is one of the important metaheuristic algorithms which mimic the natural tendency of HW (humpback wheel). The bubble net strategy is the main attraction of the WOA algorithm. The original algorithm is very useful for solving continuous problems. To make it appropriate and useful in the binary system, Vijay Kumar and Dinesh Kumar proposed a binary version of this algorithm. In the binary version for updating the position of HW (humpback wheel), they have used STF (sigmoidal transfer function). They have applied their proposed WOA algorithm on 29 benchmark functions. Their proposed WOA can be applied to solve real-life applications and EE (electrical engineering) problems [5].

The natural tendency of hunting behavior of HW (humpback whale) is imitated in WOA (whale optimization technique). Abdelazim G. Hussien and his co-workers have proposed that how the original WOA can be adapted to handle BOP (binary optimization problem). For this reason, to transfer from CSP (continuous search space) to BSP (binary search space), we have to use a V-shaped and S-shaped TF (transfer function). To illustrated the performance and functionality of their proposed BWOA (binary whale optimization algorithm), they have tried on real-world TSP (traveling salesman problem), twenty-two benchmark function, and engineering optimization problems. They also compared their algorithm with five other MOA (metaheuristic optimization algorithms). They have shown that BWOA performs better than other MOA [6].

In a dark environment, the echolocation mechanism is used by the bats. Their head size is small, and they extract high-frequency sound that helps them to differentiate the sound level in the echolocation mechanism. Rock Z. Shi and Timothy K. Horiuchi implemented bat's difference processing in the VLSI model. They have used a neural unit and a circuit model for the mathematical calculation to capture the level difference. They have also done the functional model of the system [7].

To solve the optimization problem efficiently, BA (bat algorithm) is one of the effective metaheuristic algorithms in recent days. Gulnur Yildizdan and his co-workers propped an improved version BA (bat algorithm), which will enhance the exploration and exploitation part of BA (bat algorithm). In their proposed algorithm, they 
implemented this BA algorithm in individuals, and the selection has been made on the probability value. It is calculated by using the algorithm. To check the performance, they have implemented it on the CEC 2011 realworld problem, CEC 2005 benchmark function, large-scale CEC 2010 benchmark function, and so on [8].

In image segmentation, multilevel thresholding is an important operation. By selecting optimal thresholding for the exhaustive search method is computationally very expensive. So, Xiofeng Yue and Hongbo Zhang have proposed Modified HBA (hybrid bat algorithm). They have used SIW (smart inertia weight), BA (bat algorithm), and GCO (genetic crossover operation) to select OT (optimal threshold). They have compared and evaluated their result using various threshold levels [9].

\section{Theoretical Background}

\subsection{WOA (Whale Optimization Algorithm):}

First, Mirjalili and Lewis proposed WOA (whale optimization algorithm) in 2016. WOA is a nature-inspired MOA (metaheuristic optimization algorithm) technique. The social behavior of HW (humpback wheel) is mimicked in WOA. HW (humpback wheel) is considered as a type of social animal. Their hunting behaviors are defined as the GHM (group hunting method). At the time of hunting the prey, they used a bubble net in their hunting strategy. HW (humpback wheel) makes their GHS (group hunting strategy) such a way that feeding their younger ones and finding the prey is advantageous. At the first step of hunting, they try to search for a group of prey. After locating the prey, they drive deep into the water. Then this HW (humpback wheel) generates bubbles, and the prey is forced to move into the bubble-like net. This type of hunting strategy is called bubble-net feeding. This type of strategy is used by HW (humpback wheel) to catch a large group of fish. It may be possible that the fish size is small. HW tries to find small size fish due to two reasons. The first one is that this big size HW (humpback wheel) has no teeth. The second reason that their throat size is very small. If the size of the fish is small, then they can engulf the whole fish. The bubble net strategy of HW is mathematically expressed and graphically depicted in the algorithm in the next few subsequent sections. Image fusion can be efficiently optimized using the WOA algorithm in medical imaging [10-12]. The strategy of hunting the prey by HW (humpback wheel) is divided into two parts. The first part is the exploration and the second part is exploitation. These two main parts are divided into three subparts. The first one is SP (searching for prey). The second subpart is BNF (bubble net feeding), and the last part is EP (encircling the prey) [13].

\section{A. Encircling the Prey:}

The hunting process of HW (humpback wheel) is divided into two parts first one is the exploration, and this part again divided into two subparts. Fist subpart is encircling the prey. The HW (humpback wheel) first observe the location of the prey carefully. After that, they start to encircle the prey. At the starting of the hunting process, many search agents are deployed in WOA. Each individual SA (search agent) provides a solution. Best SA (search agent) is selected depending on the fitness value. On the basis of the global best result, HW (humpback whale) modifies their position. To realize the encircling behavior of HW (humpback wheel), it is necessary to go through the mathematically model, which is depicted below [14].

$$
\begin{aligned}
& \vec{D}=\left|\vec{C} \cdot \overrightarrow{X^{*}}(t)-\vec{X}(t)\right|---------(1) \\
& \vec{X}(t+1)=\overrightarrow{X^{*}}(t)-\vec{A} \cdot \vec{D}
\end{aligned}
$$

The number of iteration is denoted by 't'. The coefficient vector is represented by $\vec{A}$ and $\vec{C}$. Vector $X^{*}$ gives the global solution of WOA. The position vector is indicated by $\vec{X}$. Here element by element multiplication has been done, and the element by element multiplication is denoted by ' $'$ 'After each iteration, WOA gives an improved value of $X^{*}$ which moves towards an accurate solution. Coefficient vector $\vec{A}$ and $\vec{C}$ is mathematically depicted below.

$$
\begin{aligned}
& \vec{A}=2 \cdot \vec{a} \cdot \vec{r}-\vec{a}- \\
& \vec{C}=2 \cdot \vec{r}
\end{aligned}
$$

In each iteration step, a's value is decreased from 2 to 0 . The range of random vector $\vec{r}$ is $[0,1]$.The whale can update their old position to a new position by using the mathematical equation (1). 
Journal of Soft Computing Paradigm (JSCP) (2020)

Vol.02/ No.04

Pages: 195-208

http://irojournals.com/jscp/

DOI: https://doi.org/10.36548/jscp.2020.4.001

\section{B. Bubble Net Attacking and Feeding:}

The second subpart of exploration is bubble-net attacking. The picture of the bubble net tracking is depicted below.

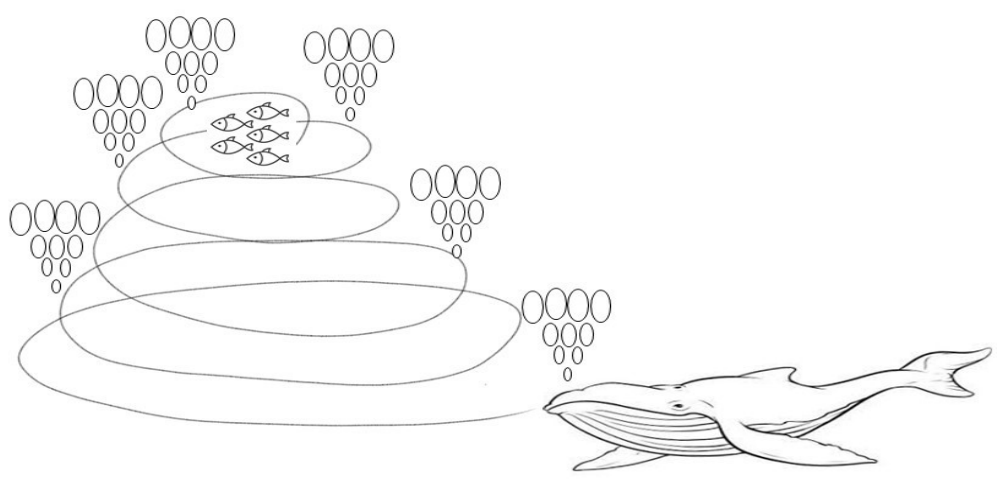

Figure 2. Bubble net hunting behavior of humpback wheel

The procedure of the bubble net by HW (humpback wheel) is divided into two mechanisms.

\section{a) SES (Shrinking Encircling System):}

In this mechanism of SES the $\vec{a}$ 's value decreases from 2 to 0 iteratively. Three number equation has been built in a way that value of $\vec{A}$ is reduced by $\vec{a}$. It can also be said that random vector $\vec{A}$ ranges from -a to +a that is [-a, + a]. In every subsequent step, a's value is decreased from two to zero. The search agent of the WOA algorithm moves to the best location from the original location by modifying the value of $\vec{A}$ in the range $[-1,1]$.

\section{b) SSUP (Spiral Shaped Updating Position):}

The HW (humpback wheel) first finds the prey location. Depending upon the location of the prey, HW calculates the distance from the prey for each individual HW. The HW tries to move near the prey using the special movement. If the position of HW is $(X, Y)$ and the position of the prey is $\left(X^{*}, Y^{*}\right)$ then the behavior of HW and the special movement can be uniquely depicted by using logarithmic movement. The special algorithmic path modifies the position of HW (humpback wheel). This natural behavior of HW (humpback wheel) is mathematically listed in the equation below.

$$
\begin{aligned}
& \overrightarrow{D^{\prime}}=\left|\overrightarrow{X^{*}}(t)-\vec{X}(t)\right| \\
& \overrightarrow{X^{\prime}}(t+1)=\overrightarrow{D^{\prime}} \cdot e^{b l} \cdot \cos (2 \pi l)+\overrightarrow{X^{*}}(t)
\end{aligned}
$$

Distance between prey and i th HW (humpback wheel) is defined as $\overrightarrow{D^{\prime}}=\left|\overrightarrow{X^{*}}(t)-\vec{X}(t)\right|$ (by considering the best solution). The value of ' 1 ' is any random value, which ranges between $[-1,1]$. Here ' $b$ ' is described as a constant, and it is used to define LPM (logarithmic spiral movement). After locating the prey position, HW (humpback wheel) drives deeper into the water. So, that they can make a circle outside the position of the prey. Then the best fitness valued HW (humpback wheel) moves spirally toward the prey. Equation six or equation two is used to update the position of HW (humpback wheel). They may or may not be updated, and the probability of updating the position is $50 \%$, which is represented mathematically in the equation below [15].

$$
\begin{gathered}
\vec{X}(t+1)=\overrightarrow{X^{*}}(t)-\vec{A} \cdot \vec{D} \quad \text { if } p<0.5 \\
\overrightarrow{D^{\prime}} \cdot e^{b l} \cdot \cos (2 \pi l)+\overrightarrow{X^{*}}(t) \quad \text { if } p>0.5---(7)
\end{gathered}
$$

A random number ' $p$ ' ranges [0,1]. HW 's (Humpback wheel) hunting strategy is divided into two interesting strategies. The first one is BNS (bubble net strategy), and the second strategy is SB (searching Behaviour), which they use for searching the prey. It is mathematically depicted in the WOA algorithm [16]. The position is updated according to the equations in each iteration, and this is depicted below. 


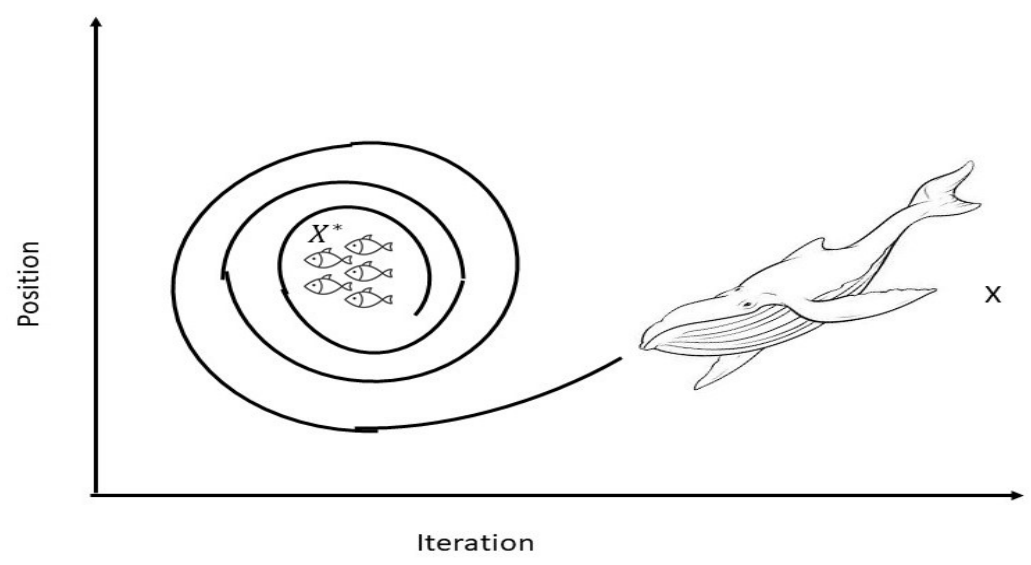

Figure 3. Whale position upgradation using spiral movement

\section{C) Search for Prey:}

The movement of HW (humpback wheel) is linear, and their searching process is also linear. In this segment, searching-space for hunting the prey is the main attention for us. The humpback wheel first drives to the furthest location from each other. So, that they can find the prey. In the exploration part, a vector $\vec{A}$ is used in a mathematical equation to scan the prey position. If the vector $\vec{A}$ 's value is more than +1 or less than -1 , then they continue their search. Global optima of the WOA algorithm is determined when in the mathematical equation, the A's value is $|\vec{A}|>1$. This criterion is used to avoid local optima. Then the position is updated mathematically by the equation below.

$$
\begin{aligned}
& \vec{D}=\left|\vec{C} \cdot \overrightarrow{X_{\text {rand }}}-\vec{X}\right| \\
& \vec{X}(t+1)=\overline{X_{\text {rand }}}-\vec{A} \cdot \vec{D} \text {------(9) }
\end{aligned}
$$

Random vector $\overrightarrow{X_{\text {rand }}}$ is selected depending on the position of SA (search agent). Each individual position of HW (humpback whale) in the WOA algorithm is generated randomly. In each iteration from worst positioned, HW (humpback wheel) updates their position with respect to the best whale in the pack or with respect to other agents. Parameter ' $a$ ' is controlled by LSS (linear search strategy). The a's value is reduced linearly from 2 to 1 . This is represented mathematically in $|a|>1$. Another way we can say that exploration happens when $|a|>1$ and exploitation happen when $|a|<1$ [17].

\subsection{BA (Bat Algorithm):}

In 2010, Yang and his co-workers proposed BA (bat algorithm), which is a metaheuristic algorithm. Bat uses echolocation in the dark environment to locate their nest, detect the prey, and avoid obstacles [18]. BA algorithm does not stop until they find a suitable solution. Bats runs with velocity $v_{i}$ from the position $x_{i}$. At that time with varying wavelength $\lambda$, loudness is $A_{0}$, and their velocity is $f_{\min }$. Bat hunts the prey with a random search strategy. First, in the algorithm, the pulse frequency is set, which can vary in the range $r \in[0,1]$. Bat varies the pulse frequency according to the distance from the prey. At first, a loudness value $A_{0}$ is taken. This loudness value changes in between minimum $A_{\min }$ and maximum $A_{0}$. The range of frequency is $\left[f_{\min }, f_{\max }\right]$ and the range of wavelength is in between $\left[\lambda_{\min }, \lambda_{\max }\right]$ [19]. The finding technique of prey in the bat algorithm is depicted below. 


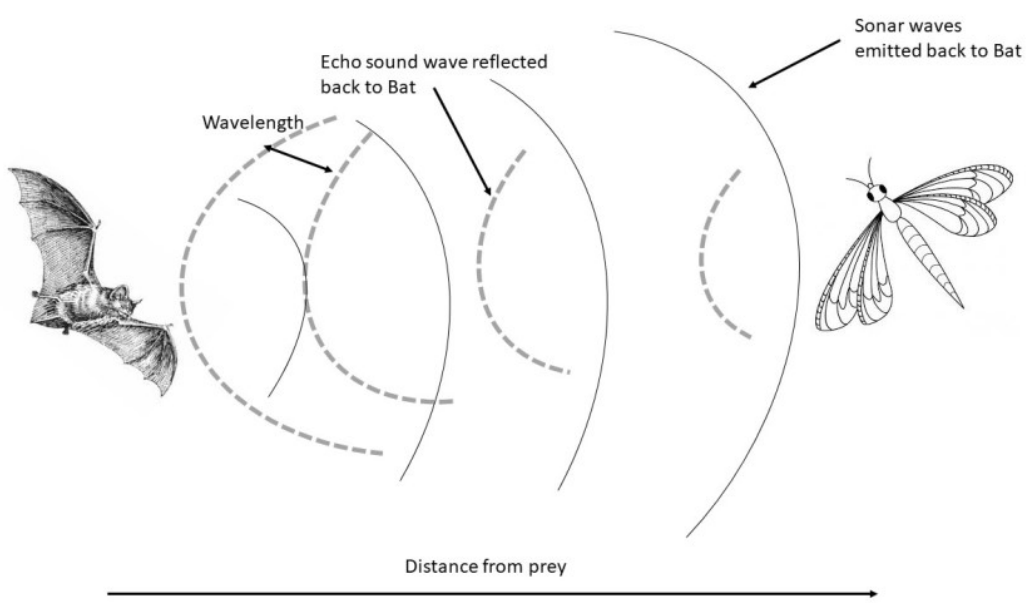

Figure 4. Bat algorithm prey finding technique by echolocation

At the time of testing, the wavelength value of BA can be any value. The wavelength value in the BA algorithm is very important. Generally, frequency ' $\mathrm{f}$ ' and wavelength ' $\lambda$ ' are related to each other, and it can change at any time. The range of frequency $\mathrm{f}$ is defined $\left[0, f_{\max }\right]$. In the $\mathrm{BA}$ algorithm, the frequency value is set to lower value when bats travel at a further distance. For that reason, the wavelength value is lower. When the bat travels a shorter distance, then the scenario is the opposite. At that time, the frequency is higher as well as the wavelength is also higher. In the BA algorithm, wavelength and frequency play a crucial role. The reason is that they are related to computational cost and exploration capacity. The pulse rate varies in the range [0,1]. Zero defines, there is no pulse rate, and one defines the maximum pulse rate [20].

At the starting position of bats are initialized as $x_{i}(\mathrm{i}=1,2, \ldots, \mathrm{n})$. In this case, the velocity is $v_{i}$ in $\mathrm{n}$-dimension space, which changes in consecutive stages. After the ' $t$ ' time unit, a new solution is generated where $x_{i}^{t}$ denotes the new position and $v_{i}^{t}$ denotes the velocity. Then the new equations are mathematically depicted below [21].

$$
\begin{aligned}
& f_{i}=f_{\text {min }}+\left(f_{\text {max }}-f_{\text {min }}\right) \beta \text {-------------(10) } \\
& v_{i}^{t}=v_{i}^{t-1}+\left(x_{i}^{t}-x_{*}\right) \cdot f_{i} \\
& x_{i}^{t}=x_{i}^{t-1}+v_{i}^{t}
\end{aligned}
$$

Here $\beta$ defines the uniformly distributed random vector whose range is defined as $\beta \in[0,1] . x_{*}$ defines the global best solution among ' $\mathrm{n}$ ' number of bats. Velocity is significantly increased by $\lambda_{i} f_{i}$. The new velocity is settled by using $\lambda_{i}$ (or $f_{i}$ ). For doing so, we have first to fix the parameter $f_{i}$ (or $\left.\lambda_{i}\right)$. The frequency value is used either $f_{\max }=$ 100 or $f_{\min }=0$, and it depends on the problem. In the beginning, a random frequency is taken, and the range of frequency that it changes is $\left[f_{\min }, f_{\max }\right]$. Naturally, the walking of bats in a random fashion and at the end, a solution is generated by each bat. The best solution is taken at the end of those solutions in LSP (local search process). This behavior is mathematically depicted in the equation below [22-23].

$$
x_{\text {new }}=x_{\text {old }}+\in A^{t}
$$

The range for random value $\in$ is [-1,1]. All the bats produce some loudness, and the calculated loudness value for this is $A^{t}=<A_{i}^{t}>$. Position and velocity are updated for all bats after each iteration, and the process is the same as the other PSO algorithms. Space of movement and rage is controlled by the frequency $f_{i}$. At the end of each iteration loudness $A_{i}$ and pulse emission rate $r_{i}$ is calculated. Loudness is decreased when the bat is very nearer to the prey. The general value of $A_{0}$ is 100 , and the value of $A_{\min }$ is 1 . At the time bat find the prey then the $A_{\min }$ value decreased to 0 , and the bat stops the emission of the sound [24-25].

$$
A_{i}^{t+1}=\propto A_{i}^{t}, r^{t+1}=r_{i}^{0}\left[1-e^{\gamma t}\right]
$$

Here $\gamma$ and $\propto$ is denoted as a constant. 
Journal of Soft Computing Paradigm (JSCP) (2020)

Vol.02/ No.04

Pages: 195-208

http://irojournals.com/jscp/

DOI: https://doi.org/10.36548/jscp.2020.4.001

\subsection{LSA (Local search Algorithm):}

After getting the best individual, the LSS algorithm is used in each iteration. So, we can find a better individual. Binary values are 0 or 1 for each search agent and other position of those search agents, and these binary values are changed. For a new search agent, the value is modified in every move, and according to the old search agent position, the new search agent moves to the position. This natural tendency happens when a better one is replaced by the best one. The probability of happening in those situations is very small [26]. In our proposed MWOA, this type of general and distinctive characteristic is happened and is obtained by using LSS algorithms. Incorporating the LSS algorithm in our proposed MWOA algorithm, two steps need to be included. The first step that needs to be introduced is FS (feature segmentation), and the second step is the PM (partial movement). Local search operation in the LSS algorithm has been improved by using two different operators [27]. One of the operators is 'Deletion,' and the other one operator is 'addition'. The addition operator has been implemented for selecting the desired number of features. The deletion operator has been used to delete the desired number of features from a particular position. It can be said in another way that for the addition of dissimilar products, add operator is used. The main objective of the LSS algorithm is to choose a distinct feature in such a way that the correction should be lower [28-29].

\section{Proposed Modified Whale Optimization Algorithm:}

The WOA is MOA(metaheuristic optimization algorithm) algorithm based on that out MWOA (modified whale optimization algorithm) algorithm is proposed. Like other straight forward metaheuristic optimization techniques, WOA also suffers trapping of local minima and premature convergence. Our proposed MWOA (modified WOA) is free from such drawbacks. To built our proposed MWOA, we have used the LSA (local search algorithm), WOA (whale optimization algorithm), and BA (bat algorithm).In the main whale optimization algorithm, up to parameter updation of I, P, a, C, and A are copied to MWOA ( 1 to $6^{\text {th }}$ line of the algorithm depicted that). Depending on the value of $p$, the searching process changes. If the $p$ value is below 0.5 unit, then the searching process is done using circular encircling (line 7 in the algorithm depict that). The local search algorithm has been used if the A's range is from -1 to +1 of a unit radius circle (line 8 in the algorithm depict that). Here in the LSA (local search algorithm) test case has been taken from zero, and it continued up to 100 times. Here, in that case, it may be possible that an unknown object may be more than one. So, for flexibility of searching up to 100 nearest unknown objects has been taken consideration, and the worst unknown object, which is compared by fitness value, is taken for first consideration (line 8.1 to 8.17 in the algorithm depict that). For searching, the worst affected cell LSS algorithm has been used. After that result is stored, and equations are updated depending on the worst unknown object (line 9 in the algorithm depict that). First, the searching algorithm searches for one unit radius, and if it is unsuccessful in finding the unknown object, then another searching process has been used to find the unknown object beyond one unit radius. Bat algorithm has been used here to find the unknown object if the object is beyond one unit radius. BA (bat algorithm) has an advantage; it can find the object easily in the dark environment. The searching process of the bat algorithm used a sound repulsion technique. For this reason, a sound probe can be used effectively. First, a random frequency is chosen. When the first repulsion happened, at that time-frequency and wavelength has been updated successfully. The velocity of the sound probe increase or decreases. Depending on that, the sound probe stops its movement when the worst unknown object is found (lines 10.1 to 10.6 in the algorithm depict that). Equation 9 is updated when the affected cell location has been found. This type of searching is very effective and efficient and gives an extra layer of protection inside the human body to find the unknown object. The spiral search optimization technique has been used when the value of $p$ is more than or equal to 0.5 units. After that, equation six is updated. This process is continued to the last iteration. At the end of the process, the best-fused image has been obtained that is used for clinical diagnosis in medical imaging.

Algorithm. Modified Whale Optimization Algorithm (MWOA)

1. First, a random population of CS is generated $X_{i}(i-1,2,3, \ldots . ., n)$

2. The fitness value is calculated for each CS probe;

3. $X^{*}$ is the best CS probe

4. while $(\mathrm{t}<$ \#iterations) do

5. for each CS probe do

6. a,C,P,A and I are updated in this stage

7. $\quad$ if $(p<0.5)$ then

$8 . \quad$ if $(|\mathrm{A}|<1)$ then

To test the case first set $S=\emptyset$

for each search coverage $\mathrm{C}$ do 
Journal of Soft Computing Paradigm (JSCP) (2020)

Vol.02/ No.04

Pages: 195-208

http://irojournals.com/jscp/

DOI: https://doi.org/10.36548/jscp.2020.4.001

8.3
8.4
8.5
$\sum$ of $N_{c}$
8.6

selected.

8.7

8.8

8.9

8.10

8.11

8.12

8.13

8.14

9.

10

10.1

10.2

10.3

10.4

10.5

10.6

10.7

10 to 13

10.8

10.9

10.10

10.11

10.12

10.13

10.14

10.15

10.16

11.

12.

13.

14.

15.

16.

17.

18.

19.
Find start camera probe, $N_{s}$ repeat the process

100 neearest neighbour are found by adding one step into

Comparing among 100 neighbours best fitness neighbour is

If it is seen that best fitness neighbour is usefull then

else $N_{c} \leftarrow$ the best fitness neighbour

end if

break

until the value of $\mathrm{C}$ is satishfied or the maximum iteration is reached;

end for $S=S \cup\left(\sum\right.$ of $\left.N_{c}\right)$

Position of the probe (CS probe) is updated (Equation 1); else if $(|A| \geq 1)$ then

the objective function $f(X), X=\left(X_{1}, \ldots ., X_{d}\right)^{T}$;

Population of SP (sound probe) is initialized as $X_{i}(i=1,2, \ldots, n)$ and $v_{i}$;

Pulse frequency is $f_{i}$ which is at $X_{i}$;

Pulse rate and loudness is initialized as $r_{i}$ and $A_{i}$ respectively;

While ( $\mathrm{t}<$ \#iterations) do

New solution is generated by setting the frequency

Velocity and solution/location is updated by using the equation from

if $\left(\right.$ rand $\left.>r_{i}\right)$ then

A solution is selected;

A local solution is generated;

A new solution is generated by flying randomly;

if $\left(\right.$ rand $\left.<A_{i}\right) \&\left(f\left(X_{i}\right)<f\left(X_{*}\right)\right)$ then

The solution is accepted;

$r_{i}$ is increased and $A_{i}$ is decreased in this step;

Find the best $X_{*}$;

The global best solution is recorded from SP (sound probe).

Select an agent $X_{\text {rand }}$ which is the best result $X_{*}$;

Position of CS probe is updated by using the equation 9 ;

else if ( $p \geq 0.5$ ) then

Position of CS probe is updated by using the equation 6;

Each CS probe is validated and go beyond search space;

Fitness value of each CS probe is calculated;

Update $X^{*}$ in case a better image and solution is observed;

$\mathrm{t}++$;

return $X^{*}$.

Proposed modified WOA algorithm's flow diagram is drawn in the figure below

ISSN: 2582-2640 (online)

Submitted: 25.07.2020

Accepted: 29.08.2020

Published: 07.09.2020 
Journal of Soft Computing Paradigm (JSCP) (2020)

Vol.02/ No.04

Pages: 195-208

http://irojournals.com/jscp/

DOI: $\underline{\text { https://doi.org/10.36548/jscp.2020.4.001 }}$

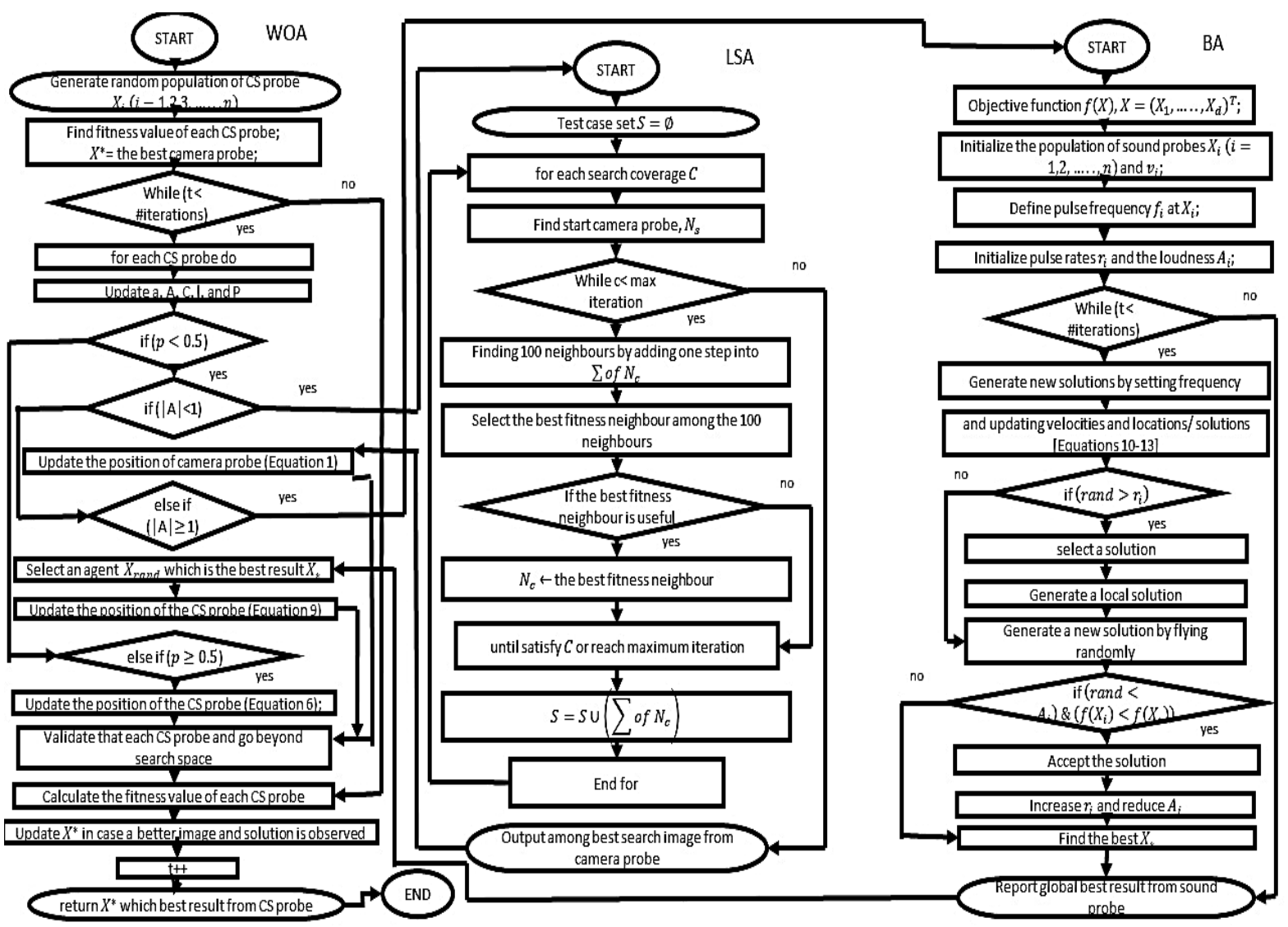

Figure 5. Proposed Modified Whale Optimization Algorithm

\section{Experimental Results:}

Our own algorithm has been compared with other metaheuristics optimization techniques like WOA, CS-GWO, GWO, and CS optimization algorithm. For this reason, the key features of those algorithms are used. A comparison has been made in two steps. In the first step, the setting parameter is listed in table 1 before performing the algorithm. In the second step, table 2 defines the implementation result. Table 3 describes the comparison percentage of optimization, and table 4 depicts the comparison result. For this purpose, we have used the key features of those nature-inspired algorithms.

Table 1. Setting of parameters

\begin{tabular}{|c|c|c|c|c|c|c|}
\hline $\begin{array}{c}\text { Description of } \\
\text { parameters }\end{array}$ & $\begin{array}{c}\text { MWOA } \\
\text { parameter } \\
\text { setting }\end{array}$ & $\begin{array}{c}\text { WOA } \\
\text { Parameter } \\
\text { setting }\end{array}$ & $\begin{array}{c}\text { CS-GWO } \\
\text { parameter } \\
\text { setting }\end{array}$ & $\begin{array}{c}\text { GWO } \\
\text { parameter } \\
\text { setting }\end{array}$ & $\begin{array}{c}\text { CS parameter } \\
\text { setting }\end{array}$ & $\begin{array}{c}\text { Unselected } \\
\text { parameter } \\
\text { setting }\end{array}$ \\
\hline $\begin{array}{c}\text { Size of } \\
\text { population }\end{array}$ & 40 & 40 & 40 & 40 & 40 & 40 \\
\hline $\begin{array}{c}\text { Dimension of } \\
\text { problem }\end{array}$ & 40 & 40 & 40 & 40 & 40 & 40 \\
\hline $\begin{array}{c}\text { The maximum } \\
\text { iteration that } \\
\text { has been made }\end{array}$ & 500 & 500 & 500 & 500 & 500 & 500 \\
\hline $\begin{array}{c}\text { Total runs that } \\
\text { have been made }\end{array}$ & 40 & 40 & 40 & 40 & 40 & 40 \\
\hline
\end{tabular}

Before starting the algorithm, the parameter of MWOA should be initialized correctly. So, In the above table, the setting parameter is described. First, in the column description of the parameter is defined, and the setting parameter of MWOA, CS-GWO, WOA, GWO, CS, and the unselected algorithm is defined in the consecutive column. In the second row, the size of the population is described under the description of the parameter. The size of the parameter is taken 40 in all cases. In the third row dimension of the population is initialized under the column of the description of the parameter where the dimension of the problem is taken as 40 in each case. Next 
Journal of Soft Computing Paradigm (JSCP) (2020)

Vol.02/ No.04

Pages: $195-208$

http://irojournals.com/jscp/

DOI: https://doi.org/10.36548/jscp.2020.4.001

to that row, a maximum iteration that has been made is listed under the first column, and the maximum iteration is made 500. In the end row, the total run in a runtime of the algorithm is mentioned, and that is 40 in each case.

Table 2. The result after implementation of MWOA, CS-GWO, GWO, CS, and Unselected algorithm.

\begin{tabular}{|c|c|c|c|c|c|c|}
\hline & MWOA & CS-GWO & WOA & GWO & CS & Unselected \\
\hline $\begin{array}{c}\text { Number of } \\
\text { initial positions }\end{array}$ & 200 & 240 & 440 & 440 & 440 & 1600 \\
\hline $\begin{array}{c}\text { Number of } \\
\text { initial } \\
\text { populations }\end{array}$ & 40 & 40 & 40 & 40 & 40 & 40 \\
\hline $\begin{array}{c}\text { The ratio of } \\
\text { cross over }\end{array}$ & Dynamic & Dynamic & Dynamic & Dynamic & Dynamic & Static \\
\hline $\begin{array}{c}\text { Ration of } \\
\text { mutation }\end{array}$ & Dynamic & Dynamic & Dynamic & Dynamic & Dynamic & Static \\
\hline
\end{tabular}

After applying the algorithm, the result of MWOA is noted. So, in the above table, the result after the implication is described. Here we have listed MWOA as well as other competitive algorithms (CS-GWO, WOA, GWO, CS, and unselected algorithm) results in the consecutive column. In the second row number of the initial position is described under table 2. For MWOA, WOA, and GWO, the number of initial positions is 200, 440, and 440, respectively. The number of the initial position for CS-GWO and CS algorithms is 240 and 440, respectively. Whereas for an unselected algorithm is 1600 . In the third row, the number of initial population is listed. The number of initial population is taken 40 in each case. Next to that, the ratio of cross over is mentioned where MWOA, CS-GWO, WOA, GWO, and CS, the ratio of cross over is dynamic, but for an unselected algorithm, it is static. In the end row, the ration of mutation is listed. For MWOA, CS-GWO, WOA, GWO, and CS, the ratio of mutation is dynamic, whereas it is static for an unselected algorithm.

Table 3. Comparison of percentage of optimization covered by MWOA against CS-GWO, WOA, GWO, CS, and Unselected algorithm.

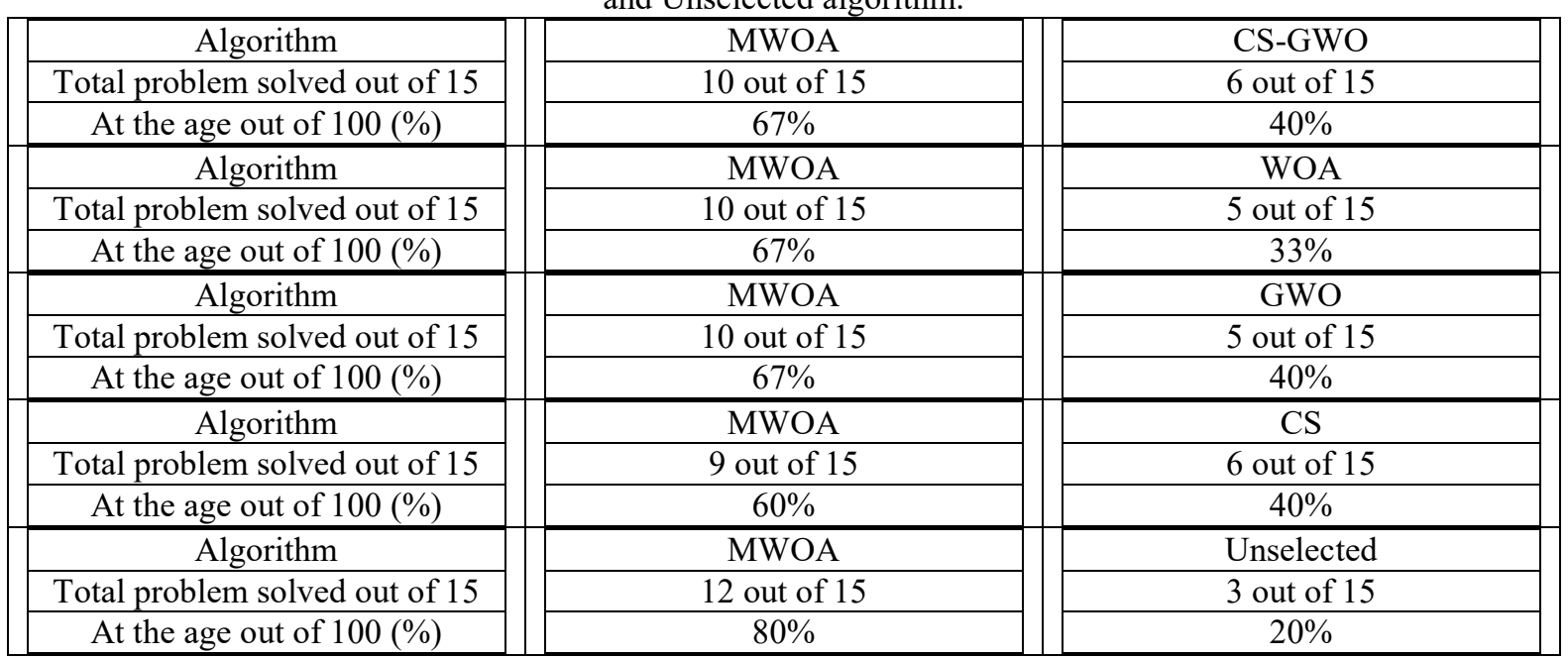

To understand the superiority of MWOA than the other nature-inspired algorithm, a detailed discussion is needed. In this regard, the comparison of MWOA against CS-GWO, WOA, GWO, CS, and unselected algorithm is depicted in table 3. In this table, a comparison is made in terms of the percentage of optimization covered. Firstrow MWOA and CS-GWO algorithms are compared. The total problem was solved by MWOA 10 out of 15 problems. At the same time, CS-GWO can only solve 6 out of 15 problems. If it is converted in terms of percentage, then MWOA is $67 \%$ coverage, which is superior to CS-GWO. That can only cover $40 \%$ coverage. Then we have compared MWOA with WOA. Here we can see MWOA can solve ten problems out of 15 problems, whereas WOA can only solve five problems out of 15 problems. If we describe in terms of percentage, then MWOA can cover $67 \%$, and WOA can cover $33 \%$, which is very less. In the next row, GWO is compared with the MWOA. Here MWOA solved ten problems out of 15 problems, whereas GWO solved only five problems out of 15 problems. So, MWOA can make 67\% coverage compare to $33 \%$ coverage in GWO. Next to that, MWOA is compared with the CS algorithm. Here MWOA solved nine problems out of 15 problems. That is $60 \%$ coverage. 
Journal of Soft Computing Paradigm (JSCP) (2020)

Vol.02/ No.04

Pages: $195-208$

http://irojournals.com/jscp/

DOI: https://doi.org/10.36548/jscp.2020.4.001

At the same time, CS can solve six problems out of 15 problems. That is $40 \%$ overall coverage. In the last row, the comparison of MWOA and an unselected algorithm is listed. In that bounded time, MWOA solved 12 problems out of 15 problems, whereas an unselected algorithm can solve only three problems out of 15 problems. That can be represented in terms of percentage for easy understanding. The MWOA can make $80 \%$ percent problem coverage compare to $20 \%$ problem coverage using an unselected algorithm.

The result of comparison among GWO, MWOA, WOA, CS, and CS-GWO is listed in the table below.

Table 4. Comparative Results

\begin{tabular}{|c|c|c|c|c|}
\hline $\begin{array}{c}\text { Algorithm } \\
\text { Name }\end{array}$ & $\begin{array}{c}\text { Original Features } \\
\text { Number }\end{array}$ & $\begin{array}{c}\text { Subsets Features } \\
\text { Number }\end{array}$ & Accuracy & Best Fitness \\
\hline MWOA & 40 & 5 & $87.13 \%$ & $83.327 \%$ \\
\hline CS-GWO [30] & 40 & 6 & $83.54 \%$ & $78.811 \%$ \\
\hline WOA & 40 & 11 & $83.54 \%$ & $75.613 \%$ \\
\hline GWO [30] & 40 & 11 & $83.54 \%$ & $75.561 \%$ \\
\hline CS [30] & 40 & 11 & $83.54 \%$ & $75.261 \%$ \\
\hline $\begin{array}{c}\text { Unselected } \\
{[30]}\end{array}$ & 40 & 40 & $77.15 \%$ & $55.107 \%$ \\
\hline
\end{tabular}

First, we have implemented MWOA. Then we have compared our proposed MWOA with unselected, CS, GWO, WOA, and CS-GWO algorithms. So, there should not any doubt about the supremacy of MWOA. In the above table, we have compared the result of MWOA with other nature-inspired algorithms. In the first column, algorithm names are titled. In the next column, the Original feature number is grouped. Next to that substate feature number is depicted. Then the accuracy of the different algorithms is listed. In the last column, the fitness percentage is recorded. In the above table, it is visible that the sixth one is the worst one because here, no optimization technique has been used. If we use only the CS algorithm, then it gives a better result without using any optimization technique. In terms of fitness percentage, GWO gives a better result than CS. GWO and WOA are the same in terms of accuracy. If we consider fitness WOA is better than GWO. CS-GWO gives more acceptable results than CS, GWO, and WOA, but in terms of fitness and accuracy, MWOA gives the best result. In terms of accuracy, MWOA is $4.297 \%$ better than CS-GWO, which is the closest one and 5.73\%supirior to CS-GWO if we consider in terms of fitness.

Our proposed MWOA (modified whale optimization algorithm) is incorporated with BA, and the LSA algorithm is more optimal in contrast to GWO, CS-GWO, WOA, and CS.

\section{Conclusions:}

In this article, a novel metaheuristic algorithm of a modified whale optimization algorithm has been proposed. Different efficient metaheuristic and heuristic algorithms have been used for optimizing image fusion. To increase the searching efficiency of our proposed MWOA algorithm, BA (bat algorithm) and LSA (local search algorithm) have been used. This BA (bat algorithm) and LSA (local search algorithm) optimize the algorithm in such a way that it provides the best accurate result of image fusion. The main problem of original WOA is that trapping of local minima and premature convergence. Here in our proposed MWOA, overcome those problems by judicious design strategy. The whole algorithm has been improved, and approximation is minimized by using BA, WOA, and LSA algorithms. In the result and discussion, we have compared MWOA against CS-GWO, WOA, GWO, $\mathrm{CS}$, and unselected algorithms. In the first table, it is noticeable that for the shake of simplicity, we have taken the same size of the population, dimension of the problem, the maximum iteration, and total runs. In table 2 , the implementation result has been shown. Depending upon the subset of feature number, the number of initial positions has changed. Table 3 shows the excellency of MWOA over CS, CS-GWO, GWO, WOA, and unselected algorithms in terms of problem coverage of specific time-bound. Table 4 shows the supremacy of MWOA over CS, CS-GWO, GWO, WOA, and unselected algorithm in terms of in term of accuracy and fitness percentage. The quality of the proposed architecture is successfully assessed by simulation using ModelSim and also on-board testing in the case of real-time application. 
Journal of Soft Computing Paradigm (JSCP) (2020)

Vol.02/ No.04

Pages: 195-208

http://irojournals.com/jscp/

DOI: https://doi.org/10.36548/jscp.2020.4.001

\section{References:}

[1] Abdel-Basset, M., Chang, V., \& Mohamed, R. (2020). HSMA_WOA: A hybrid novel Slime mould algorithm with whale optimization algorithm for tackling the image segmentation problem of chest X-ray images. Applied Soft Computing, 106642.

[2] Yin, B., Wang, C., \& Abza, F. (2020). New brain tumor classification method based on an improved version of whale optimization algorithm. Biomedical Signal Processing and Control, 56, 101728.

[3] Luo, J., He, F., \& Yong, J. (2020). An efficient and robust bat algorithm with fusion of opposition-based learning and whale optimization algorithm. Intelligent Data Analysis, 24(3), 581-606.

[4] AlJame, M., Ahmad, I., \& Alfailakawi, M. (2020). Apache Spark Implementation of Whale Optimization Algorithm. Cluster Computing, 1-14.

[5] Kumar, V., \& Kumar, D. (2020). Binary whale optimization algorithm and its application to unit commitment problem. Neural Computing and Applications, 32(7), 2095-2123.

[6] Hussien, A. G., Hassanien, A. E., Houssein, E. H., Amin, M., \& Azar, A. T. (2020). New binary whale optimization algorithm for discrete optimization problems. Engineering Optimization, 52(6), 945-959.

[7] Liu, L., Luo, S., Guo, F., \& Tan, S. (2020). Multi-point shortest path planning based on an Improved Discrete Bat Algorithm. Applied Soft Computing, 106498.

[8] Yildizdan, G., \& Baykan, Ö. K. (2020). A novel modified bat algorithm hybridizing by differential evolution algorithm. Expert Systems with Applications, 141, 112949.

[9] Yue, X., \& Zhang, H. (2020). Modified hybrid bat algorithm with genetic crossover operation and smart inertia weight for multilevel image segmentation. Applied Soft Computing, 90, 106157.

[10] Gautam, A., \& Biswas, M. (2018, June). Whale Optimization Algorithm Based Edge Detection for Noisy Image. In 2018 Second International Conference on Intelligent Computing and Control Systems (ICICCS) (pp. 1878-1883). IEEE.

[11] Ahmed, A. S., Attia, M. A., Hamed, N. M., \& Abdelaziz, A. Y. (2017, December). Comparison between genetic algorithm and whale optimization algorithm in fault location estimation in power systems. In 2017 Nineteenth International Middle East Power Systems Conference (MEPCON) (pp. 631-637). IEEE.

[12] Dao, T. K., Pan, T. S., \& Pan, J. S. (2016, November). A multi-objective optimal mobile robot path planning based on whale optimization algorithm. In 2016 IEEE 13th International Conference on Signal Processing (ICSP) (pp. 337-342). IEEE.

[13] Emary, E., Zawbaa, H. M., \& Salam, M. A. (2017, September). A proposed whale search algorithm with adaptive random walk. In 2017 13th IEEE International Conference on Intelligent Computer Communication and Processing (ICCP) (pp. 171-177). IEEE.

[14] Kumawat, I. R., Nanda, S. J., \& Maddila, R. K. (2017, November). Multi-objective whale optimization. In Tencon 2017-2017 ieee region 10 conference (pp. 2747-2752). IEEE.

[15] Sharawi, M., Zawbaa, H. M., \& Emary, E. (2017, February). Feature selection approach based on whale optimization algorithm. In 2017 Ninth International Conference on Advanced Computational Intelligence (ICACI) (pp. 163-168). IEEE.

[16] Xu, H., Fu, Y., Fang, C., Cao, Q., Su, J., \& Wei, S. (2018, September). An Improved Binary Whale Optimization Algorithm for Feature Selection of Network Intrusion Detection. In 2018 IEEE 4th International Symposium on Wireless Systems within the International Conferences on Intelligent Data Acquisition and Advanced Computing Systems (IDAACS-SWS) (pp. 10-15). IEEE.

[17] Zhang, C., Fu, X., Peng, S., \& Wang, Y. (2018, May). Linear unequally spaced array synthesis for sidelobe suppression with different aperture constraints using whale optimization algorithm. In 2018 13th IEEE Conference on Industrial Electronics and Applications (ICIEA) (pp. 69-73). IEEE.

[18] Azlan, N. A., \& Yahya, N. M. (2019, March). Modified Adaptive Bats Sonar Algorithm with Doppler Effect Mechanism for Solving Single Objective Unconstrained Optimization Problems. In 2019 IEEE 15th International Colloquium on Signal Processing \& Its Applications (CSPA) (pp. 27-30). IEEE.

[19] Chaudhary, R., \& Banati, H. (2018, September). Modified shuffled multi-population bat algorithm. In 2018 International Conference on Advances in Computing, Communications and Informatics (ICACCI) (pp. 943-951). IEEE.

[20] Li, M., Liu, X., Li, R., Zheng, R., \& Zhao, W. (2018, May). Fault Diagnosis of Transformer Based on Chaotic Bats Algorithm Optimizing Fuzzy Petri Net. In 2018 2nd IEEE Advanced Information Management, Communicates, Electronic and Automation Control Conference (IMCEC) (pp. 885-889). IEEE.

[21] Savsani, P., Jhala, R. L., \& Savsani, V. J. (2014). Comparative study of different metaheuristics for the trajectory planning of a robotic arm. IEEE Systems Journal, 10(2), 697-708.

[22] Senthilnath, J., Kulkarni, S., Benediktsson, J. A., \& Yang, X. S. (2016). A novel approach for multispectral satellite image classification based on the bat algorithm. IEEE Geoscience and Remote Sensing Letters, 13(4), 599-603.

ISSN: 2582-2640 (online) 
Journal of Soft Computing Paradigm (JSCP) (2020)

Vol.02/ No.04

Pages: $195-208$

http://irojournals.com/jscp/

DOI: https://doi.org/10.36548/jscp.2020.4.001

[23] Singh, D., Salgotra, R., \& Singh, U. (2017, March). A novel modified bat algorithm for global optimization. In 2017 International Conference on Innovations in Information, Embedded and Communication Systems (ICIIECS) (pp. 1-5). IEEE.

[24] Gan, J. E., \& Lai, W. K. (2019, June). Automated Grading of Edible Birds Nest Using Hybrid Bat Algorithm Clustering Based on K-Means. In 2019 IEEE International Conference on Automatic Control and Intelligent Systems (I2CACIS) (pp. 73-78). IEEE.

[25] Luthra, I., Chaturvedi, S. K., Upadhyay, D., \& Gupta, R. (2017, April). Comparative study on nature inspired algorithms for optimization problem. In 2017 International Conference of Electronics, Communication and Aerospace Technology (ICECA) (Vol. 2, pp. 143-147). IEEE.

[26] Lara, A., Sanchez, G., Coello, C. A. C., \& Schutze, O. (2009). HCS: A new local search strategy for memetic multiobjective evolutionary algorithms. IEEE Transactions on Evolutionary Computation, 14(1), 112-132.

[27] Martins, S. L., Resende, M. G., Ribeiro, C. C., \& Pardalos, P. M. (2000). A parallel GRASP for the Steiner tree problem in graphs using a hybrid local search strategy. Journal of Global Optimization, 17(1-4), 267-283.

[28] Moradi, P., \& Gholampour, M. (2016). A hybrid particle swarm optimization for feature subset selection by integrating a novel local search strategy. Applied Soft Computing, 43, 117-130.

[29] Ameur, M. S. B., \& Sakly, A. (2017). FPGA based hardware implementation of Bat Algorithm. Applied Soft Computing, 58, 378-387.

[30] Xu, H., Liu, X., \& Su, J. (2017, September). An improved grey wolf optimizer algorithm integrated with Cuckoo Search. In 2017 9th IEEE international conference on intelligent data acquisition and advanced computing systems: technology and applications (IDAACS) (Vol. 1, pp. 490-493). IEEE.

\section{Authors Biography:}

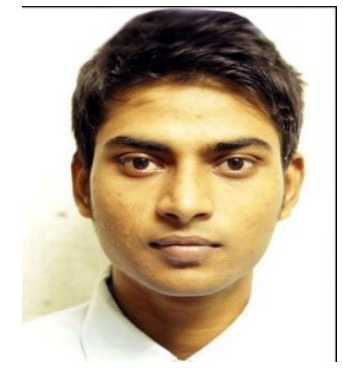

Sayantan Dutta received his BTech degree in Electronics and Communication Engineering form Meghnad Saha Institute of Technology in 2016. He has completed his MTech from Indian Institute of Engineering Science and Technology, Shibpur in 2020. His research interest is synthesizable digital VLSI circuit design, Nature inspired algorithm, Optimization process, VLSI physical design and architecture for medical imaging and satellite imaging.

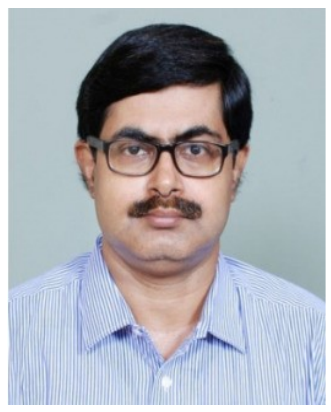

Ayan Banerjee is presently working as the Head of the Department of Electronics and Telecommunication Engineering at Indian Institute of Engineering Science and Technology (IIEST), Shibpur, India. He obtained B.E. in 1994 from Bengal Engineering College, Shibpur, Calcutta University and M. Tech. from IIT, Kharagpur in Electronics and Electrical Communication Engineering with specialization in Integrated Circuits and Systems Engineering in 1999. He completed his Ph.D. from IIT, Kharagpur in 2013 successfully. His research area includes Digital Signal/ Image Processing, VLSI Architectures for real-time signal processing systems etc. 